\title{
Werner Syndrome Protein-Unwinding Function to Explain Disease
}

\author{
Raymond J. Monnat Jr. and Yannick Saintigny
}

(Published 31 March 2004)

\begin{abstract}
Werner syndrome (WS) is one of three heritable human genetic instability/cancer predisposition syndromes that result from mutations in a member of the gene family encoding human RecQ helicases. Cellular defects are a prominent part of the WS phenotype. Here we review recent work to identify in vivo functions of the WS protein and discuss how loss of function leads to cellular defects. These new results provide clues to the origin of cell lineage-specific defects in WS patients and suggest a broader role for Werner protein function in determining disease risk in the general population.
\end{abstract}

\section{Introduction}

Werner syndrome (http://sageke.sciencemag.org/cgi/content/ full/sageke;2001/1/ns2) (WS) is growing up. After an uncertain childhood that included almost a half century of neglect (1), research on WS has entered a vigorous adolescence. Much of the continuing interest in this autosomal recessive disease (caused by loss-of-function mutations in the $W R N$ http://sageke. sciencemag.org/cgi/genedata/sagekeGdbGene;189 gene) has focused on the appearance of premature aging in association with an elevated risk of age-associated diseases such as cancer, atherosclerotic cardiovascular disease, diabetes mellitus, and osteoporosis in affected individuals (2-4). The presumption is that a deeper understanding of WS will provide useful new information about the pathogenesis of these clinically important, age-associated disease processes, as well as useful new insights into more general aspects of the biology of human aging $(5,6)$.

Our focus in this Review is on functions of the WRN protein (http://sageke.sciencemag.org/cgi/content/full/2002/13/re2), a DNA helicase, at the cellular level. Our aim is to indicate how loss of function promotes cell- and lineage-specific defects in vivo.

Genetic instability is an important consequence of the loss of WRN function and one of the first abnormal phenotypes to be identified in cells from WS patients (7). Genetic instability after the loss of WRN function is a plausible "intermediate" phenotype for experimental analyses, because it identifies an immediate consequence of the loss of WRN function at the cellular level. This phenotype also provides a conceptually useful way to think about the origins of the lineage-specific defects observed in affected individuals. Thus, we consider how genetic instability might arise in the absence of WRN function and the consequences of genetic instability in specific cell lineages during and after development. Also discussed are emerging data that indicate a larger role for WRN function in human health and disease than is suggested by the rari-

\footnotetext{
Raymond J. Monnat Jr. is in the Departments of Pathology and Genome Sciences at the University of Washington, Seattle, WA 98195, USA. Yannick Saintigny is at the Laboratoire d'Etude de la Recombinaison, Département de Radiobiologie et Radiopathologie, Commissariat à l'Energie Atomique, 92265 Fontenay aux Roses Cedex, France. E-mail: monnat@u.washington.edu (R.J.M.)
}

ty of the WS clinical phenotype.

We emphasize in this discussion the role of WRN in homologous recombination (HR). Homologous recombination, as suggested by its name, involves the exchange of genetic information between homologous (identical) DNA sequences in the genome. In germ cells, these exchanges promote the reshuffling of genetic information between generations, whereas in somatic cells, HR promotes DNA repair and the successful completion of DNA replication. The recent identification of a role for the WRN protein in HR in human somatic cells was unexpected. A reconsideration of molecular, biochemical, and cytological data on WS in the context of HR begins to explain mechanistic links among recombination, cell viability, and mutagenesis in WS cells. The most surprising aspect of this story is the conclusion that WS disease pathogenesis might be driven by a recombination defect. This conclusion is the opposite of what has been widely assumed-that WS is a hyperrecombination syndrome - and thus of particular heuristic value. The assumption that WS is a hyperrecombination syndrome came initially from the identification of chromosomal rearrangements and extensive deletions in WS cells and cell lines (see below), and the assumption that these genomic rearrangements were the result of excessive recombination. As we discuss below, the deletion mutator phenotype appears to be a consequence of defective, not excessive, HR. A few of the most important aspects of the WRN gene, WRN protein, and WS phenotype are summarized below to begin this discussion. Readers are also referred to recent reviews in SAGE KE [see Fry Review (http://sageke.sciencemag. org/cgi/content/full/sageke;2002/13/re2) and Cheng Perspective (http://sageke.sciencemag.org/cgi/content/full/sageke;2003/31/p e22)] and in print that provide additional details and viewpoints on many of the topics discussed here $(4,8-13)$.

\section{The WRN Gene and Protein}

Positional cloning of the WRN (http://sageke.sciencemag.org/ cgi/genedata/sagekeGdbGene;189) gene in 1996 provided a strong stimulus for research on in vivo function and disease pathogenesis. The $W R N$ locus, situated at chromosomal position 8p12, encodes a $162-\mathrm{kD}$ member of the human RecQ helicase family (14). The five members of this family all possess 3 '-to- 5 ' helicase activity as well as adenosine triphosphatase activity $(13,15)$. WRN is unique among the human RecQ helicases in possessing an additional 3 '-to-5' exonuclease activity (16-18) (Fig. 1).

The biochemical similarity of human RecQ helicases to homologs identified in prokaryotes (http://sageke.sciencemag.org/ cgi/content/full/2003/40/nw137) and single-celled eukaryotes suggested the potential for functional parallelism and helped to identify several model organisms in which to investigate RecQ helicase function [reviewed in $(12,13)]$. Potential roles for the human RecQ helicases in genome stability assurance were emphasized by the identification of three human RecQ helicase deficiency syndromes: WS, Bloom syndrome (caused by muta- 
tions in the $B L M \mathrm{http}: /$ sageke.sciencemag.org/cgi/genedata/ sagekeGdbGene; 8 gene), and a subset of Rothmund-Thomson syndrome. In each syndrome, genetic instability and a predisposition to neoplasia result from the loss of function of a different human RecQ helicase protein $(14,19-21)$ (see "A Jump-Start for Replication" http://sageke.sciencemag.org/cgi/content/ abstract/2002/13/nw45).

Analyses of WRN activities on defined nucleic acid substrates indicate that WRN can unwind and/or degrade several types of DNA metabolic intermediates. These include three- and four-way DNA junctions and gapped, branched, or unpaired DNA regions [reviewed in $(10,22)]$. DNA molecules containing three- or four-stranded junctions arise as part of DNA replication, as a consequence of DNA repair, and during HR. The increasingly rich body of in vitro biochemical data on WRN has been complemented by protein interaction studies that indicate physical and/or functional cross-talk between WRN and general nucleic acid metabolic proteins such as replication protein A (http:// sageke.sciencemag.org/cgi/content/full/sageke;2002/13/re2) and more specialized proteins involved in DNA synthesis, recombination, or repair $(10,22)$. The $W R N$ mutations identified in WS patients all truncate and promote loss of the WRN protein from patient cells (23-26). The absence of WS-associated missense mutations that selectively inactivate either the WRN exonuclease or helicase activity is noteworthy: This finding suggests that it is the loss of the helicase and exonuclease functions of WRN that leads to WS. We discuss this point further below in light of new experimental results.

\section{The Importance of Cellular Phenotype}

Three consistent cellular defects have been identified after the loss of WRN function. This triad includes (i) cell proliferation defects that have been best defined in the fibroblast cell lineage $(27,28)$; (ii) selective sensitivity to a small number of DNAdamaging agents (29-33); and (iii) genetic instability (observed at both the cytogenetic and molecular genetic level) in different cell lineages in vivo and in vitro (34-36). These aspects of the WS cell phenotype are experimentally tractable, can be easily quantified, and appear to directly reflect WRN function. Thus, cellular phenotype has been an important focus in attempts to understand WRN function.

Analysis of WRN function at the cellular level has also been conceptually important as a way to integrate and interrelate the molecular, biochemical, cytogenetic, and cytological data on WRN function. Further, understanding WRN function at the cellular level is likely to be directly relevant to disease pathogenesis. Thus, an important goal and milestone for current WS research is to develop a sophisticated molecular-level model of WRN function at the level of single cells that is both quantitative and predictive.

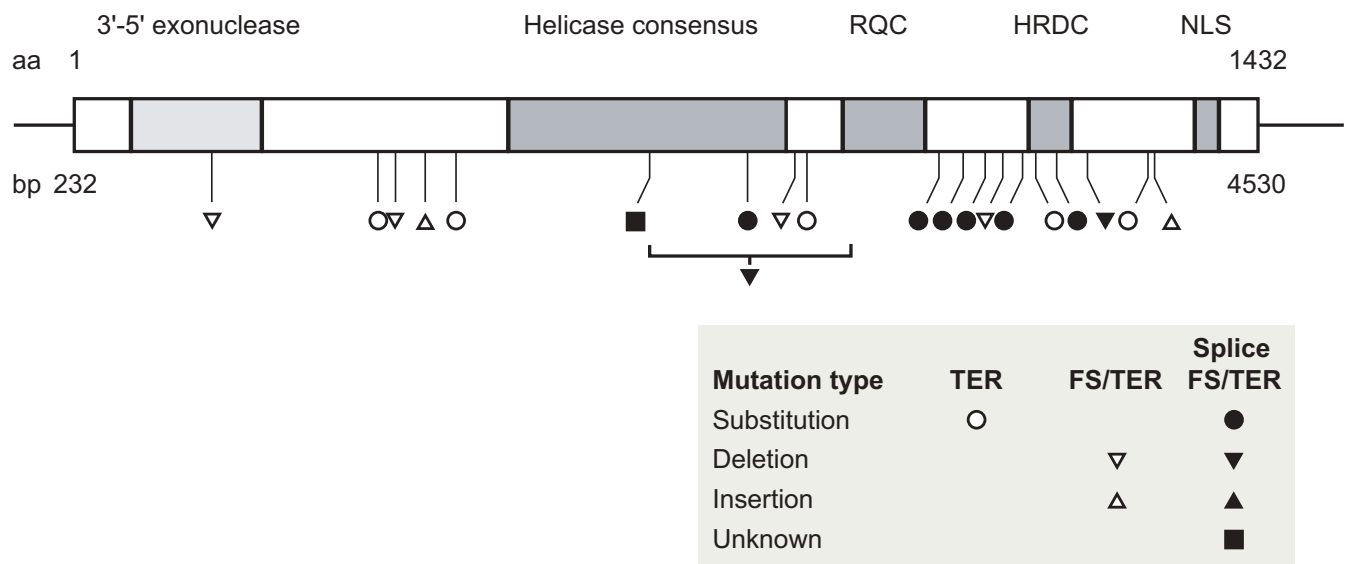

Fig. 1. Domain structure of WRN and spectrum of WS-associated WRN mutations. The central box indicates the WRN open reading frame with amino acid residue numbering indicated on top and cDNA base (ive protein motifs are indicated by shaded boxes and labels: (i) case and RNaseD-C-terminal (HRDC) domain and the C-terminal nuclear localization signal (NLS). The positions and molecular types of mutations identified in WS patients are indicated by symbols below. All mutations thus far identified truncate the WRN open reading frame and cause loss of the nuclear local-

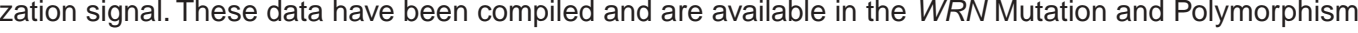
Database (www.pathology.washington.edu/research/werner/ws_wrn.html), developed and maintained at the University of Washington. TER, termination; FS, frameshift; splice FS/TER, mutations that interfere with splicing and lead to frameshifts with downstream stop codon(s) in the new reading frame.

\section{WRN Function in HR}

The past 5 years have seen a resurgence of interest in HR in human somatic cells (37-40). This renewed interest has been driven by several factors, including (i) data indicating the quantitative importance of HR in mammalian DNA break repair [see, for example, (41)]; (ii) the critical role played by HR in mammalian development $(42,43)$ and cell viability (44); and (iii) data from bacteria and single-celled eukaryotes that indicate a critical role for HR proteins and HR function in DNA repair and the rescue of stalled replication forks (45-47). A final stimulus for renewed interest in mammalian HR comes from the fields of human genetics and genomics: An increasing number of human diseases are being identified that might represent defects in recombination [reviewed in $(48,49)$ ], and a better understanding of HR might provide more efficient ways to modify human genes for therapeutic gain. Moreover, many of the proteins mediating human HR have polymorphic variants with the potential to affect expression and/or function, and several variants have already been associated with disease risk, most notably for neoplasia $(48,50)$.

Although there have been hints for some time that WRN might play a role in HR [see, for example, (51)], the nature of this involvement only became clear when the behavior of "direct repeat" recombination reporter (DR reporter) substrates was examined in WS and control cells (Fig. 2) (52). The use of 
this type of chromosomally integrated substrate allows the frequency and rate of spontaneous and damage-induced recombination to be quantified and the molecular nature of recombinants to be analyzed to provide mechanistic insight into mammalian recombination pathways $(40,53,54)$.

Two classes of genetically active recombinant molecules can be recovered after DR reporter plasmids undergo recombination: (i) conversion-type recombinant molecules, in which the DR reporter is intact and one of the two reporter alleles has been converted to an active form; and (ii) crossover or "popout" molecules, in which a single active allele remains with the loss of intervening DNA. Either or both recombinant classes can be recovered depending on the structure of the initial or substrate DR reporter plasmid. These two types of recombinant molecules can be generated by several different pathways (Fig. 2). Human and other mammalian cells show a clear preference for the generation of conversion-type events, the most conservative of the potential outcomes that generate active reporter alleles $(53,55-57)$.

Analyses of spontaneous HR in WS cells using this type of DR reporter have revealed a 25 -fold reduction in the rate of generation of viable recombinant daughter cells that retain growth potential as compared with normal cells. This reduction is observed despite an apparently normal rate of generation of recombinant molecules in WS cells. Related to this finding is a reduction in the proportion of viable recombinant cells that harbor conversiontype recombinant molecules (52). This WS-associated HR defect can be rescued by expression of catalytically active WRN protein or by the expression of the RusA bacterial resolvase protein, suggesting that WRN, like RusA, functions in the resolution of HR products. Resolution or postsynapsis is the stage of HR when successful recombinant molecules are disentangled and segregated to daughter cells. The reduced cell survival that accompanies the WS HR defect can also be suppressed by dominant negative RAD51 (http://sageke.sciencemag.org/cgi/ genedata/sagekeGdbGene;121), the key protein involved in nucleoprotein filament formation, strand invasion, and the search for homology in the early stages of HR (58) (Fig. 3). Expression of this dominant negative form inhibits HR. The simplest explanation for these results is that HR initiation is normal in WS cells, but a portion of the products of RAD51-dependent HR cannot be successfully resolved in the absence of WRN function.

Recent data have allowed us to take this story one step further and to address the roles of the WRN exonuclease and helicase functions in HR and in cell survival after DNA damage. By expressing WRN proteins in which single amino acid substitutions were used to inactivate the WRN exonuclease and/or helicase functions, we showed that both catalytic activities were essential for WRN to function in HR resolution. To our surprise, single missense mutant forms of WRN that lacked exonuclease or helicase activity supported normal cell survival levels after DNA damage in the absence of HR. The WS cell phenotypes of an HR defect and reduced cell survival after DNA damage did not differ between WS cells that lacked detectable WRN or that expressed catalytically inactive WRN at physiological levels $(58,59)$.

These results indicate that catalytic functions [as opposed to postulated scaffolding functions (http://sageke.sciencemag.org/ cgi/content/full/2003/31/pe22)] of WRN are critical for HR, and that HR and cell survival can in some cases be separated. Our results thus differ from recently published work by Chen et al. proposing an important structural role for WRN in determining the outcome of break repair events $(60)$. We think the most like-

Fig. 2. DR reporter plasmid structure and recombinant classes. Structure of a DR reporter plasmid, pNeoA (57). Open arrows indicate direct repeat neomycin phosphoavy lines indicating the region of homology between linker insertion sites. Two major (he outcome of recombination pathways porter allele $\left(n e o^{+}\right)$that allows growth in the presence of the drug G418, a neomycin analog. The two outcomes are distinguished by the structure of the resulting recombinant molecule and by whether cells also retain or lose resistance to hygromycin (hygr, (a) restance cassette; G418', G418-sensitive; G418', G418-resistant). Gene or via single-ended invasion and synthesis-dependent strand annealing. Noncrossover

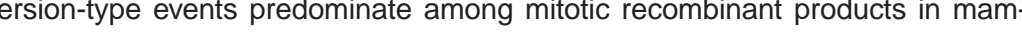
trachromosomal recombination, unequal sister chromatid exchange, or single-strand annealing. These recombination pathways and the genetic requirements for each are discussed in detail in $(40,54,116)$.

ly explanation for this discrepancy is Chen et al.'s use of fibroblasts immortalized by expression of the catalytic subunit of telomerase, hTERT (http://sageke.sciencemag.org/cgi/ genedata/sagekeGdbGene;205). Although hTERT expression suppresses the growth defect of primary WS fibroblasts, as does the SV40 T antigen expressed in the cells used in our experiments (61-64), hTERT also appears to suppress other important aspects of the WS cellular phenotype, such as selective drug sensitivity and the WS HR defect. The expression of hTERT also appears to alter the outcome of some classes of repair event in WS cells, such as plasmid rejoining [see (65)], that is likely to depend on nonhomologous DNA end joining (http://sageke. sciencemag.org/cgi/content/full/2003/8/re3) (NHEJ), a repair pathway in which DNA ends are joined without regard for the presence of homologous DNA sequences.

An important conclusion from our experimental results is that both of the WRN catalytic activities need to be lost in order to generate the WS cellular phenotype. This conclusion pro- 
vides a satisfying explanation for the spectrum of WRN mutations in WS patients that, as noted above, lack missense mutations that selectively inactivate the WRN exonuclease or helicase activities. The identification of a requirement for both WRN catalytic activities in HR also raises the interesting prospect that inherited or somatically acquired $W R N$ missense mutations could selectively cripple the WRN HR function without appreciably affecting cell viability. Such mutations, if they are segregating in the human population, would likely confer a phenotype different from WS [see (59) for further discussion].

\section{Resolution and Its Consequences}

A role for WRN in the resolution of HR products was unexpected. Successful resolution is important if HR products are to be topologically disentangled and accurately segregated to generate viable recombinant daughter cells $(66,67)$. In addition to topologically disentangling recombinant DNA duplexes, resolution determines the proportion of crossover and noncrossover recombinants. Crossover recombinants have the potential to promote gene or chromosomal rearrangement and the loss of genetic information. Thus, not surprisingly, the generation of different types of resolution products is under tight control, with mammalian HR strongly favoring the generation of noncrossover products (40).

How the resolution of recombinant molecules is managed at the molecular level is best understood in prokaryotes. Successful resolution appears to involve at least three discrete steps: (i) the recognition and binding of DNA molecules containing recombination junctions such as Holliday junctions or D loops; (ii) branch migration of the DNA junctions; and (iii) junction cleavage at or near crossover points, followed by ligation of the now-separated DNA molecules to form intact recombinant DNA duplexes (46, 66-68). In Escherichia coli, a dedicated molecular machine known as the RuvABC complex binds, branch-migrates, and cleaves Holliday junction-containing products at crossover points to give rise to different classes of recombinant DNA molecules (68).

The comparable proteins that mediate resolution in eukaryotes have been intensively sought and are now just beginning to come into focus (67). Two human RecQ helicases, WRN and BLM, can bind and branch-migrate model recombination substrates such as Holliday junctions, one of the desired activities for a resolution complex $(69,70)$. The exonuclease activity of WRN might also be useful for resolving certain types of recombinant products such as D loops (Fig. 4A). However, neither WRN nor BLM can cleave model recombination junctions in vitro, and neither appears to be present in mammalian cell frac- tions that possess the ability to branch-migrate and cleave model HR junction substrates $(71,72)$.

Some types of eukaryotic recombination junctions might be cleaved and resolved by the heteromeric protein Mus81Eme1/Mms4. This endonuclease, first identified in parallel analyses in budding and fission yeast, consists of two proteins that interact to form a structure-specific endonuclease [reviewed in $(67,73)]$. Human homologs exist for both proteins, and both proteins appear to be involved in recombination and the response to DNA damage. For example, Mus81 contributes to one of the recombination resolution activities identified by biochemical fractionation of mammalian cell extracts $(72,74)$. Human Mus81 localizes to sites of DNA damage in the nuclei of HeLa cells and colocalizes in the nucleolus with WRN and

Fig. 3. Model of WRN function in HR. DNA damage, replication, or repair can initiate HR (left). WRN promotes HR resolution or replication restart to ensure cell viability and genetic stability $\left(\mathrm{WRN}^{+}\right.$arrow). In the absence of $\mathrm{WRN}\left(\mathrm{WRN}^{-}\right), \mathrm{HR}$ resolution and/or replication restart fails, shown in ovals: Reexpressing WRN protein (+wt WRN) improves both cell survival and the recov(+RusA). The dependence of WRN phenotypes on RAD51 pathway function and products can be revealed by expressing a dominant negative form of mammalian RAD51 protein (+SMRAD51) that suppresses mitotic recombination in WRN and controls cells while improving WRN cell survival after cis-Pt-induced damage. Anticipated consequences of survival in the absence of HR function are mutagenesis and genetic instability (58).

BLM (75). Moreover, depletion of Mus81 mRNA by RNA interference leads to a reduction in the generation of recombinants (in the same recombination reporter cell lines that were originally used to identify the HR defect in WS cells) and a loss of cell viability $(52,76)$.

A recent extensive analysis of murine Eme1 indicates that it is part of a structure-specific endonuclease with a preference for $3 \rho$ flap substrates. This type of DNA structure, in which a nicked DNA duplex contains a single-stranded DNA tail that is displaced at the nick, can arise in the context of DNA replication or DNA repair. Murine embryonic stem (ES) cells that lack Eme1 are sensitive to DNA cross-linking agents such as cisplatin (cis-Pt) and mitomycin-C, and exhibit an increased incidence of damage-dependent sister chromatid exchange and elevated levels of both spontaneous and damage-induced chromosomal aberrations as compared with wild-type ES cells (77). 
These and other data indicate that the Mus81-Eme1/Mms4 endonuclease is a plausible candidate for one of the eukaryotic resolution endonuclease activities, despite uncertainty as to the likely in vivo substrates and the quantitative importance of this resolution pathway (67).

The recent identification of RAD51C, one of the mammalian RAD51 paralogs, as a candidate resolution protein is intriguing (78). The RAD51 paralogs were originally identified on the basis of sequence conservation and the ability to complement the x-ray sensitivity displayed by certain mutant hamster cell lines. These proteins clearly play a role in the maintenance of genome stability and might have functional roles in one or more repair pathways, although the mechanistic details remain obscure (79). RAD51C appears to be part of at least two different heteromeric paralog complexes. One of these complexes, RAD51C-XRCC3, is important for both HR resolution and the replication of damaged DNA (78-81). Precisely how RAD51C acts to promote resolution, despite apparently lacking branch migration or endonuclease activity (78), is puzzling and points to the participation of RAD51C-XRCC3 in one or more resolution complexes. The number, composition, and substrate preference of these resolution complexes may be rapidly discovered, now that several participating proteins, as well as mammalian cell fractions that exhibit resolution activity, have been identified.

\section{Controlling Resolution to Suppress Gene Rearrangements}

Recent evidence indicates that RecQ helicases might also play a role in determining the proportion of crossover products in mammalian HR and in mitotic and meiotic HR in yeast $(82,83)$. Biochemical insight into one mechanism by which crossover suppression might occur in human cells was revealed in recent analyses of the resolution of recombination substrates by recombinant human BLM and DNA topoisomerase III $\alpha$ (84). This pair of proteins appears to work in concert to resolve substrates containing double Holliday junctions without the exchange of flanking markers. Junction "dissolution," as this type of resolution has been termed, thus differs from the more familiar RuvCor Mus81-like cleavage reaction discussed above $(67,84)$. These recent results parallel and extend earlier analyses of $E$. coli RecQ, in which similar catenation-decatenation activities were first described (85). One satisfying aspect of this result is that it explains one of the cytogenetic hallmarks of Bloom syndrome: the abnormally high levels of sister chromatid exchanges in cells and cell lines from BLM patients. Sister chromatid exchange crossover products arise at high frequency once crossover product formation is no longer effectively suppressed after the loss of BLM function $(40,84,86)$.

The results summarized above indicate that the WRN and BLM human RecQ he- licases might have complementary roles in suppressing gene rearrangement or loss in somatic cells. WRN appears to promote the successful resolution of HR products to favor the generation of viable conversion-type recombinants, whereas BLM acts to suppress the generation of crossover products. This model also explains why BLM and WRN, which are both envisioned to be acting in HR, have divergent loss-of-function HR phenotypes.

\section{The Role of HR in DNA Replication}

Many of the proteins that mediate HR in mammalian cells might have an additional role in insuring the completion of DNA replication (http://sageke.sciencemag.org/cgi/content/ full/sageke;2003/8/re3). The stalling or disruption of DNA replication forks appears to be common in virtually all organisms and can be accentuated by many forms of DNA damage $(45,47,87)$. The most desirable outcome of this apparently unavoidable event is to promote the successful restart of replication, while suppressing DNA breakage and genome rearrangement. DNA damage that involves both DNA strands (for example, DNA interstrand cross-links) are potent blocks to replication fork progression that may be efficiently and preferentially repaired by HR (88). Many other forms of DNA damage trigger $\mathrm{HR}$, and thus might use HR for purposes of repair or to promote damage tolerance (by, for example, lesion bypass) $(40,47)$.

One example of the latter role for HR proteins and HR func-
A

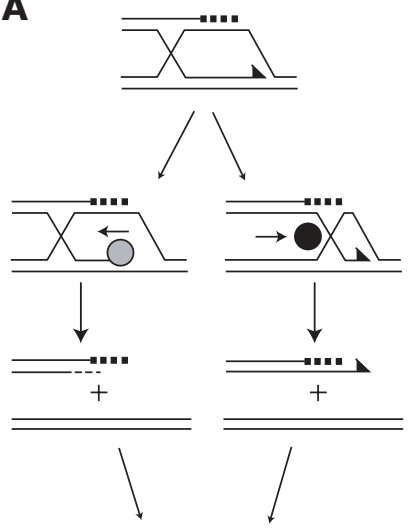

Reinvade/end join
B

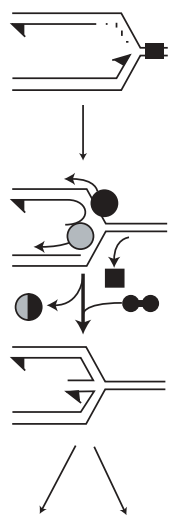

Cleave $\rightarrow$ Restart
C
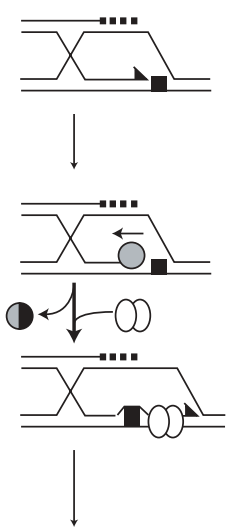

Bypass

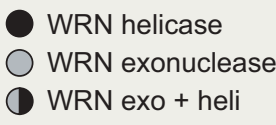

Fig. 4. Potential recombination resolution and replication restart pathways using WRN exonuclease or helicase activity. (A) Degradation or unwinding of recombination intermediates (a D loop is shown) by the WRN exonuclease or helicase activities could topologically disentangle molecules to promote resolution. (B) Degradation or unwinding of replication forks stalled by DNA damage could remove bound proteins, stabilize the fork for restart after repair, or promote regression and cleavage to generate free DNA ends. Lagging strand unwinding is shown as an example. WRN acting with RAD52 could promote a stable regressed fork that could be cleaved by Holliday junction resolvases to promote end invasion or used directly to reinitiate replication (90). (C) Disassembly or unwinding of a replication complex stalled at template DNA by WRN exonuclease (shown) or helicase activities could promote assembly of a bypass complex (open ovals) containing one or more specialized DNA polymerases. All three pathways depicted here are likely to be error-prone $(45,47,87)$. 
tion is in the processing or stabilization of stalled replication forks to either promote or inhibit the initiation of HR. Many of the postulated DNA intermediates in stalled replication forks resemble recombination intermediates, and thus might serve as substrates for recombination protein recognition and processing $(40,45,47,67,87)$. An explicit role for the E. coli RecQ protein in stalled replication fork processing has been proposed (89), and similar roles can be envisioned for WRN acting either alone or in conjunction with other proteins such as mammalian RAD52 (http://sageke.sciencemag.org/ cgi/genedata/sagekeGdbGene;122) to facilitate fork repair or stabilization (Fig. 4, B and C) (90). The WRN exonuclease activity alone, or WRN helicase in conjunction with other nuclease activities, could also act to promote the bypass of DNA damage (Fig. 4, $\mathrm{B}$ and $\mathrm{C})$.

\section{Roles for RecQs in the Cross-Talk Between HR and NHEJ}

The identification of an HR defect in WS cells provides a ready explanation for the loss of conversion-type recombinants, reduced cell viability, and selective sensitivity to DNA damaging agents that might require HR for repair (58). How, given this picture, do we make sense of reports that indicate biochemical and functional interactions between WRN and proteins that function in the NHEJ pathway, such as $\mathrm{Ku}$ and DNA-PK ${ }_{\mathrm{cs}}$ ? [See "Twisted Logic: Discoveries tangle Werner syndrome helicase story" (http:// sageke.sciencemag.org/cgi/content/ abstract/2002/12/nw40) and "Break Dancing: Werner syndrome protein might keep rowdy enzymes from doing a number on tattered DNA ends" (http://sageke.sciencemag. org/cgi/content/abstract/2002/3/nw8.)] The interrelation of WRN function, HR, and NHEJ is perhaps easiest to understand in the context of a model of substrate trafficking or flux during DNA replication (S phase) (Fig. 5).

In $\mathrm{WRN}^{+}$cells, DNA damage or S-phase intermediates that initiate HR or that require HR function are successfully converted to appropriate products. The successful resolution of these products insures high cell viability and minimizes genetic instability or gene rearrangements. In WS cells, in contrast, a portion of HR resolution events fail, giving rise to cells that undergo mitotic arrest and/or harbor DNA breaks or gaps. Such DNA breaks and gaps are substrates for recombination $(40,91)$. However, in the absence of WRN function, many of these "second tries" are again likely to fail, setting up a futile cycle that leads to mitotic death or apoptosis. DNA breaks that arise directly or indirectly from resolution failure can, in contrast, be captured and joined by NHEJ to restore chromosome integrity and insure high cell viability, albeit at the expense of mutation (92).

In this model, NHEJ function is downstream of WRN function in HR. This provides an explanation for the WRN cell phenotype, which most closely resembles an HR (as opposed to NHEJ) defect $(52,58,93,94)$. Close coordination of NHEJ and HR function in normal cells makes good teleologic sense by providing redundant ways to recognize, process, and resolve potentially dangerous DNA ends to insure chromosomal integrity and cell viability. The suggestion that WRN might be playing somewhat different roles in HR and in NHEJ, and that the balance might be altered in WRN-deficient or NHEJ-deficient cells, is also consistent with data indicating substantial crosstalk between HR and NHEJ (95-101).

Fig. 5. S-phase substrate trafficking and outcomes. As depicted in Fig. 3, several types of DNA damage, including replication fork stalling, DNA gaps that result from fork stall, and DNA breaks, can initiate HR. HR proteins can funnel these substrates into classicorrectly resolve the products to insure high cell viability and chromosomal DNA polymerases and resolvases may be able to act in concert to promote the (top) or viplication forks stalled by DNA damage either directly in the absence of HR or via HR (center; see also Fig. 4, B and C). Replication stall and gapped and

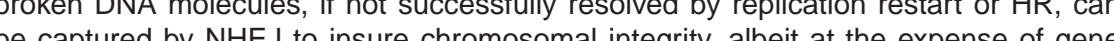
rearrangement, loss, or mutagenesis $(40,47)$. The shaded boxes to the right indicate the disposition of substrate in the presence or absence of WRN function. ining HR, cell viability, and mutagenesis data as a function of WRN status (Fig. 6). These data were all derived from the same set of well-characterized human WS and control SV40-transformed fibroblast cell lines $(52,58,102)$. Consequences of the loss of WRN function in fibroblast-lineage cells include (i) a substantial reduction in the probability of continued cell division, as measured by colony forming efficiency (CFE) or more sensitive colony size distribution (CSD) assays; (ii) a decrease in the rate of generation of recombinant daughter cells, together with the apparent loss of conversion-type recombinants; and (iii) a 10- to 100fold increase in mutagenesis at the X-linked HPRT locus (a convenient marker for examining mutagenesis, because loss-of-function mutations at this locus confer resistance to an otherwise toxic compound) $(52,58,102)$. Molecular characterization of the resulting HPRT mutations arising in WS cells indicates that they are predominantly large deletions involving the loss of from 1 to $>100$ $\mathrm{kb}$ of chromosomal DNA $(102,103)$. Most likely, these deletions originate from disrupted DNA replication intermediates or HR repair products that have been captured by NHEJ with the associated loss of intervening DNA (103).

One of the appealing aspects of the picture of WRN function 
summarized in Fig. 3, Fig. 4, and Fig. 5 is the ability to reconcile, and in part explain, many of the molecular, biochemical, cytological, and cytogenetic features of WS and of WRN function. This functional "snapshot" also begins to reveal the complexity of WRN functional roles in vivo and of functional cross-talk among the nucleic acid metabolic processes that can be influenced by WRN. The consequences of the loss of WRN function in fibroblast-lineage cells are likely to be reflected in other cell types and lineages in vivo, although this assumption needs to be documented. It will be important to replicate the quantitative and molecular data summarized in Fig. 6, using primary cells to assess how the consequences of WRN loss of function are further modified by DNA damage checkpoints and apoptosis, which can be compromised or lost in immortalized cell lines.

\section{WRN Function in Cell Lineages}

WRN and the other human RecQ helicases appear to be ubiquitously expressed, whereas HR function appears to be largely limited to dividing cells. These observations lead to an inevitable question: If the functional picture represented in Fig. 3, Fig. 4, and Fig. 5 is accurate, why are dividing cell lineages not selectively affected by the loss of WRN function? We think the most likely explanation is the following (104). All cell lineages (or at least the precursors to all cell lineages) undergo mitotic division during development. An absence of WRN function during development thus has the potential to affect all cell lineages by reducing the number of cells available for lineage construction and by "seeding" cell lineages with mutant progeny. How cell loss, genetic instability, and mutation accumulation affect a given cell lineage is likely to depend on (i) how mitotically "deep" the mature cell lineage is; (ii) how much cell editing (or loss of lineage precursor cells) occurs during and after development; (iii) whether there is redundancy of function that is lineage-specific; and (iv) how large the functional reserve is for a given lineage, tissue, or organ.

For example, the central nervous system (CNS) might be protected from the effects of loss of WRN function by virtue of stringent cell editing during development and by a comparative absence of mitotic activity during adult life. Cell editing of neuronal precursors can occur as part of normal CNS development, in which a large number of precursors are generated during the initial stages of neurogenesis, and cells in excess of the number needed to complete development undergo programmed cell death. Cell editing also appears to reflect the selective loss of precursors that lack required functional properties or are damaged. However, subtle defects might emerge over time if WRN is important for neural stem cell function during adult life (105). Conversely, cell lineages that divide continuously throughout adult life, such as those that make up the skin, gut, and bone marrow, might be tolerant of the loss of WRN function by virtue of mutation expansion-limiting lineage architecture (106), a combination of stringent cell editing and large reserves of stem or lineage repopulating cells. The cell lineages or tissues most susceptible to a loss of WRN function might be those that retain cell division potential, lack stringent cell editing during and after development, and are tolerant of and thus able to accumulate at least some types of genetic instability. The fibroblast lineage and other mesenchymal or mesodermally derived cell lineages might be selectively affected by the loss of WRN function, as suggested by the clinical and cell phenotype of WS, for precisely these reasons.

This line of reasoning leads to two important conclusions. First, we need to know more about the "normal biology" of human cell lineages before we will be able to understand and predict the in vivo consequences of loss of function of WRN or of related proteins. Second, we clearly need new and experimentally tractable cell culture and animal models in which to

$\begin{array}{lll} & \text { WRN + } & \text { WRN - } \\ \begin{array}{l}\text { Homologous recombination } \\ \text { Initiation rate }\end{array} & 10^{-4} & 10^{-4} \\ \quad \text { Viable recombinant generation rate } & 10^{-4} & 25 \text {-fold lower } \\ \quad \text { Recombinant products } & & \\ \quad \text { Conversion type } & 70 \% & 25 \% \\ \quad \text { Crossover type } & 30 \% & 75 \% \\ & & \\ \text { Cell viability } & & \\ \quad \text { Colony-forming efficiency (CFE) } & 39 \% & 24 \% \\ \quad \geq 6 \text { cell colonies } & 21 \% & 2.4 \% \\ \quad \geq 50 \text { cell colonies } & 16 \text { cells } & 4.6 \text { cells } \\ \text { Colony size distribution (CSD) } & & \\ \quad 50^{\text {th }} \text { percentile } & & 100 \text {-fold higher } \\ \quad & \sim 10^{-8} & 75 \% \\ \text { Mutagenesis } & & 25 \% \\ \quad \text { Forward mutation rate } & 39 \% & \end{array}$

Fig. 6. Quantitative aspects of the WS fibroblast phenotype. This integrated data set was developed from the same SV40-transformed fibroblast cell lines. With a loss of WRN function, the recombination initiation rate (recombinant molecules/cell doubling) is unaffected. However, the rate of successful HR resolution as measured by the rate of generation of viable recombinant daughter cells/cell doubling falls 25 -fold, together with an apparent loss of conversion-type recombinants (52). The HR defect is mirrored by a decline in cell viability in the absence of DNA damage as measured by CFE and CSD assays that measure, respectively, the generation of colonies of 6 or more or 50 or more cells (CFE), or the 50th percentile for number of cells in colonies after a defined growth interval (CSD) (58). In conjunction with the loss of viable recombinant daughter cells and reduced cell division potential, the rate of forward spontaneous mutation at the X-linked HPRT gene climbs 10- to 100 -fold per cell per generation. The predominant type of mutation identified in HPRT-deficient WS cell lines was the large deletion $[(102,103)$; see text for additional discussion]. The mitotic recombination rate is at least two orders of magnitude higher that the rate of forward HPRT mutations in $W R N^{+}$and $W R N^{-}$cells, and the recombination defect in WRN cells is accompanied by a large increase in HPRT mutagenesis. These results indicate that the recombination initiation rate is comparable in WS and control cells and that in $W R N^{+}$cells, recombination events rarely give rise to mutations. The recombination and viability deficits obersved in WS cells are further accentuated by DNA damage $(58,59)$. 
study normal lineage biology and to identify lineage-specific aspects of WRN function.

\section{WRN As a Modulator of Disease Risk in Populations}

The $W R N$ locus resembles many other human genes in that a large number of genetic variants are present in the population [see compilation and links in (24) and at the Environmental Genome Project (EGP) Web site (http://egp.gs.washington.edu)]. A subset of these are clearly disease-associated mutant alleles segregating in the human population, whereas the majority are single-nucleotide polymorphisms (SNPs) or sequence variants of uncertain functional importance. The number of these variants is surprisingly large: Recently completed resequencing of the $W R N$ exons, promoter region, and downstream untranslated region at the University of Washington as part of the EGP revealed $375 W R N$ sequence variants in 90 different DNA samples contained in the EGP's Polymorphism Discovery Resource (http://locus.umdnj. edu/nigms/products/pdr.html). These variants included a large number of SNPs in addition to nonsynonymous coding region substitutions and a previously reported nonsense mutant allele.

The potential of WRN sequence variants to modify human disease risk outside the context of WS is most clearly understood for $W R N$ heterozygotes. Heterozygous carriers of single mutant $W R N$ alleles appear to be present worldwide at frequencies ranging up to 1:100 [reviewed in (4)]. Frequency estimates of heterozygotes in the United States are in the range of $\sim 1: 250$, leading to estimates of $>10^{6}$ carriers of mutant alleles in the United States alone. WRN heterozygotes exhibit genetic instability in vivo (107), and lymphoblastoid cell lines derived from otherwise healthy $W R N$ heterozygotes display an intermediate sensitivity to killing by DNA-damaging agents that selectively kill WRN-deficient cells $(31,32)$.

Heterozygote effects have the potential to influence cancer risk or the outcome of cancer therapy. Several strategies might be helpful in establishing these links (108). For example, analysis of DNA derived from cells within clonal tumors might reveal inherited or somatically acquired $W R N$ mutations that played a role in either the origin or progression of cancer. It will be interesting to see if sporadic tumors of the types observed in WS patients $(109,110)$ are enriched for mutations or epigenetic events such as hypermethylation (http://sageke. sciencemag.org/cgi/content/full/2001/1/oa3), with associated silencing of $W R N$ expression. The identification of an aberrant or exaggerated response to chemotherapy in tumors that are heterozygous or deficient in WRN function would provide a second route to identification of a role for the WRN pathway in tumor biology. Of note, several clinically important chemotherapeutic agents such as camptothecin, mitomycin- $\mathrm{C}$, and cis-Pt selectively kill cells that are WRN-deficient or haploinsufficient $(31-33,111)$. The functional consequences of the much larger number of polymorphic $W R N$ variants and potential disease associations outside neoplasia are also just beginning to be explored $(24,112-115)$.

\section{Concluding Remarks}

WS is an example of the growing number of heritable human diseases in which a defect in genome stability assurance or genomic "housekeeping" leads to an elevated risk of one or more secondary disease phenotypes such as cancer. WS is further distinguished among the heritable housekeeping defects as one of the increasing number of human cancer predispositions that ap- pear to result from a defect in HR (49). In returning to the metaphor with which we began, all parents know that change and surprise are key features of adolescence. Our "adolescent" WS research promises an immediate future that will be challenging, surprising, and rewarding!

\section{References}

1. G. M. Martin, A brief history of research on the Werner syndrome. Gann Monogr. Cancer Res. 49, 1-10 (2001).

2. C. J. Epstein, G. M. Martin, A. L. Schultz, A. G. Motulsky, Werner's syndrome: A review of its symptomatology, natural history, pathologic features, genetics and relationship to the natural aging process. Medicine 45, 177221 (1966).

3. M. Goto, Hierarchical deterioration of body systems in Werner's syndrome: implications for normal ageing. Mech. Ageing Dev. 98, 239-254 (1997).

4. G. D. Schellenberg, T. Miki, C.-E. Yu, J. Nakura, in The Metabolic \& Molecular Basis of Inherited Disease, C. R. Scriver, A. L. Beaudet, W. S. Sly, D. Valle, Eds. (McGraw-Hill, New York, ed. 8, 2001), pp. 785-797.

5. G. M. Martin, Genetic syndromes in man with potential relevance to the pathobiology of aging. Birth Defects Original Article Ser. 14, 5-39 (1978).

6 . V. A. Bohr, Werner syndrome and its protein: clinical, cellular and molecular advances. Mech. Ageing Dev. 124, 1073-1082 (2003).

7. H. Hoehn, E. M. Bryant, K. Au, T. H. Norwood, H. Boman, G. M. Martin, Variegated translocation mosaicism in human skin fibroblast cultures. Cytogenet. Cell Genet. 15, 282-298 (1975).

8. R. M. Brosh Jr. V. A. Bohr, Roles of the Werner syndrome protein in pathways required for maintenance of genome stability. Exp. Gerontol. 37, 491 506 (2002).

9. M. Fry, The Werner syndrome helicase-nuclease-one protein, many mysteries. Sci. SAGE KE 2002, re2 (2002) (http://sageke.sciencemag.org/ cgi/content/full/sageke;2002/13/re2).

10. P. L. Opresko, W.-H. Cheng, C. von Kobbe, J. A. Harrigan, V. A. Bohr, Werner syndrome and the function of the Werner protein; what they can teach us about the molecular aging process. Carcinogenesis 24, 791-802 (2003).

11. W.-H. Cheng, V. A. Bohr, Diverse dealings of the Werner helicase/nuclease. Sci. SAGE KE 2003, pe22 (2003) (http://sageke.sciencemag.org/cgi/content/full/sageke;2003/31/pe22)

12. H. Nakayama, RecQ family helicases: roles as tumor suppressor proteins. Oncogene 21, 9008-9021 (2002)

13. I. D. Hickson, RecQ helicases: caretakers of the genome. Nat. Rev. Cancer 3. 169-178 (2003)

14. C.-E. Yu, J. Oshima, Y.-H. Fu, E. M. Wijsman, F. Hisama, S. Ouais, J. Nakura, T. Miki, G. M. Martin, J. Mulligan, et al., Positional cloning of the Werner's syndrome gene. Science 272, 258-262 (1996).

15. A. J. van Brabant, R. Stan, N. A. Ellis, DNA helicases, genomic instability, and human genetic disease. Annu. Rev. Genomics Hum. Genet. 1, 409-459 (2000).

16. S. Huang, B. Li, M. D. Gray, J. Oshima, I. S. Mian, J. Campisi, The premature aging syndrome protein, WRN, is a $3^{\prime}$ to $5^{\prime}$ exonuclease. Nat. Genet. 20, 114-116 (1998).

17. J.-C. Shen, M. D. Gray, J. Oshima, A. S. Kamath-Loeb, M. Fry, L. A. Loeb, Werner syndrome protein I: DNA helicase and DNA exonuclease reside on the same polypeptide. J. Biol. Chem. 273, 34139-34144 (1998).

18. A. S. Kamath-Loeb, J.-C. Shen, L. A. Loeb, M. Fry, Werner syndrome protein II: Characterization of the integral $3^{\prime} Æ 5^{\prime}$ DNA exonuclease. J. Biol. Chem. 273, 34145-34150 (1998)

19. N. A. Ellis, J. Groden, T.-Z. Ye, J. Straughen, D. J. Lennon, S. Ciocci, M. Proytcheva, J. German, The Bloom's syndrome gene product is homologous to RecQ helicases. Cell 83, 655-666 (1995).

20. S. Kitao, A. Shimamoto, M. Goto, R. W. Miller, W. A. Smithson, N. M. Lindor, Y. Furuichi, Mutations in RECQ4L cause a subset of cases of RothmundThomson syndrome. Nat. Genet. 22, $82-84$ (1999).

21. L. L. Wang, A. Gannavarapu, C. A. Kozinetz, M. L. Levy, R. A. Lewis, M. M. Chintagumpala, R. Ruiz-Malanado, J. Contreras-Ruiz, C. Cunniff, R. P. Erickson, et al., Association between osteosarcoma and deleterious mutations in the RECQL4 gene in Rothmund-Thomson syndrome. J. Natl. Cancer Inst. 95, 669-674 (2003).

22. C. Z. Bachrati, I. D. Hickson, RecQ helicases: suppressors of tumorigenesis and premature aging. Biochem. J. 374, 577-606 (2003).

23. M. J. Moser, J. Oshima, R. J. Monnat Jr., WRN mutations in Werner syndrome. Hum. Mutat. 13, 271-279 (1999).

24. P. W. Wagner, R. J. Monnat Jr., Werner Syndrome Mutation and Polymorphism Database, Release 2. http://www.pathology.washington.edu/research/werner/ws_wrn.html (1 July 2003).

25. M. Goto, Y. Yamabe, M. Shiratori, M. Okada, T. Kawabe, T. Matsumoto, M. Sugimoto, Y. Furuichi, Immunological diagnosis of Werner syndrome by down-regulated and truncated gene products. Hum. Genet. 105, 301-307 (1999).

26. M. J. Moser, A. S. Kamath-Loeb, J. E. Jacob, S. E. Bennett, J. Oshima, R. J. 
Monnat Jr., WRN helicase expression in Werner syndrome cell lines. $\mathrm{Nu}$ cleic Acids Res. 28, 648-654 (2000).

27. G. M. Martin, C. A. Sprague, C. J. Epstein, Replicative life-span of cultivated human cells. Effects of donor's age, tissue, and genotype. Lab. Invest. 23, 86-92 (1970)

28. T. O. Tollefsbol, H. J. Cohen, Werner's syndrome: an underdiagnosed disorder resembling premature aging. Age 7, 75-88 (1984)

29. E. Gebhart, R. Bauer, U. Raub, M. Schinzel, K. W. Ruprecht, J. B. Jonas, Spontaneous and induced chromosomal instability in Werner syndrome. Hum. Genet. 80, 135-139 (1988).

30. E. Gebhart, M. Schinzel, K. W. Ruprecht, Cytogenetic studies using various clastogens in two patients with Werner syndrome and control individuals. Hum. Genet. 70, 324-327 (1985).

31. C. E. Ogburn, J. Oshima, M. Poot, R. Chen, K. E. Hunt, K. A. Gollahon, P. S Rabinovitch, G. M. Martin, An apoptosis-inducing genotoxin differentiates heterozygotic carriers for Werner helicase mutations from wild-type and homozygous mutants. Hum. Genet. 101, 121-125 (1997).

32. M. Poot, K. A. Gollahon, P. S. Rabinovitch, Werner syndrome lymphoblas toid cells are sensitive to camptothecin-induced apoptosis in S-phase. Hum. Genet. 104 , 10-14 (1999)

33. M. Poot, J. S. Yom, S. H. Whang, J. T. Kato, K. A. Gollahon, P. S. Rabinovitch, Werner syndrome cells are sensitive to DNA cross-linking drugs. FASEB J. 15, 1224-1226 (2001)

34. D. Salk, K. Au, H. Hoehn, G. M. Martin, Cytogenetic aspects of Werner syndrome. Adv. Exp. Med. Biol. 190, 541-546 (1985).

35. M. I. Melaragno, D. Pagni, M. d. C. Smith, Cytogenetic aspects of Werner's syndrome lymphocyte cultures. Mech. Ageing Dev. 78, 117-122 (1995)

36. R. Melcher, R. von Golitschek, C. Steinlein, D. Schindler, H. Neitzel, K. Kainer, M. Schmid, H. Hoehn, Spectral karyotyping of Werner syndrome fibroblast cultures. Cytogenet. Cell Genet. 91, 180-185 (2000).

37. J. Thacker, The role of homologous recombination processes in the repair of severe forms of DNA damage in mammalian cells. Biochimie 81, 77-85 (1999).

38. L. H. Thompson, D. Schild, Homologous recombinational repair of DNA ensures mammalian chromosome stability. Mutat. Res. 477, 131-153 (2001).

39. C. Richardson, M. Jasin, Recombination between two chromosomes: implications for genomic integrity in mammalian cells. Cold Spring Harbor Symp. Quant. Biol. 65, 553-560 (2000).

40. T. Helleday, Pathways for mitotic homologous recombination in mammalian cells. Mutat. Res. 532, 103-115 (2003).

41. R. D. Johnson, M. Jasin, Sister chromatid gene conversion is a prominent double-strand break repair pathway in mammalian cells. EMBO J. 19, 3398-3407 (2000)

42. T. Tsuzuki, Y. Fujii, F. Sakumi, Y. Tominaga, K. Nakao, M. Sekiguchi, A. Matsushiro, Y. Yoshimura, T. Morita, Targeted disruption of the Rad51 gene leads to lethality in embryonic mice. Proc. Natl. Acad. Sci. U.S.A. 93, 6236 6240 (1996).

43. D.-S. Lim, P. Hasty, A mutation in mouse rad51 results in an early embryonic lethal that is suppressed by a mutation in p53. Mol. Cell. Biol. 16, 71337143 (1996).

44. E. Sonoda, M S Sasaki, J M. Buerstedde, O Bezzubova, A Shinohara, $H$ Ogawa, M. Takata, Y. Yamaguchi-Iwai, S. Takeda, Rad51-deficient vertebrate cells accumulate chromosomal breaks prior to cell death. EMBO J 17, 598-608 (1998).

45. M. M. Cox, M. F. Goodman, K. N. Kreuzer, D. Sherratt, S. J. Sandler, K. J. Marians, The importance of repairing stalled replication forks. Nature 404, 37-41 (2000).

46. A. Kuzminov, Recombinational repair of DNA damage in Escherichia coli and bacteriophage 8. Microbiol. Mol. Biol. Rev. 63, 751-813 (2000).

47. P. McGlynn, R. G. Lloyd, Recombinational repair and restart of damaged replication forks. Nat. Rev. Mol. Cell Biol. 3, 859-870 (2002).

48. A. J. Pierce, J. M. Stark, F. D. Araujo, M. E. Moynahan, M. Berwick, M Jasin, Double-strand breaks and tumorigenesis. Trends Cell Biol. 11, s52s59 (2001).

49. L. H. Thompson, D. Schild, Recombinational DNA repair and human disease. Mutat. Res. 509, 49-78 (2002).

50. B. Kuschel, A. Auranen, S. McBride, K. L. Novik, A. Antoniou, J. M. Lipscombe, N. E. Day, D. F. Easton, B. A. J. Ponder, P. D. P. Pharoah, et al., Variants in DNA double-strand break repair genes and breast cancer susceptibility. Hum. Mol. Genet. 11, 1399-1407 (2002)

51. R. Z. Cheng, S. Murano, B. Kurz, R. J. Shmookler Reis, Homologous recombination is elevated in some Werner-like syndromes but not during normal in vitro or in vivo senescence of mammalian cells. Mutat. Res. 237, 259-269 (1990)

52. P. R. Prince, M. J. Emond, R. J. Monnat Jr., Loss of Werner syndrome protein function promotes aberrant mitotic recombination. Genes Dev. 15 933-938 (2001)

53. L. B. K. Herzing, M. S. Meyn, Novel LacZ-based recombination vectors for mammalian cells. Gene 137, 163-169 (1993).

54. F. Pâques, J. E. Haber, Multiple pathways of recombination induced by double-strand breaks in Saccharomyces cerevisiae. Microbiol. Mol. Biol. Rev. 63, 349-404 (1999).

55. R. J. Bollag, A. S. Waldman, R. M. Liskay, Homologous recombination in mammalian cells. Annu. Rev. Genet. 23, 199-225 (1989).

56. R. J. Bollag, R. M. Liskay, Direct-repeat analysis of chromatid interactions during intrachromosomal recombination in mouse cells. Mol. Cell. Biol. 11, 4839-4845 (1991).

57. M. S. Meyn, High spontaneous intrachromosomal recombination rates in ataxia-telangiectasia. Science 260, 1327-1330 (1993).

58. Y. Saintigny, K. Makienko, C. Swanson, M. J. Emond, R. J. Monnat Jr., Homologous recombination resolution defect in Werner syndrome. Mol. Cell. Biol. 22, 6971-6978 (2002).

59. C. Swanson, Y. Saintigny, M. J. Emond, R. J. Monnat Jr., The Werner syndrome protein has separable recombination and viability functions. DNA Repair, in press.

60. L. Chen, S. Huang, L. Lee, A. Davalos, R. H. Schiestl, J. Campisi, J. Oshima, WRN, the protein deficient in Werner syndrome, plays a critical structural role in optimizing DNA repair. Aging Cell 2, 191-199 (2003).

61. F. S. Wyllie, C. J. Jones, J. W. Skinner, M. F. Haughton, C. Wallis, D. Wynford-Thomas, R. G. A. Faragher, D. Kipling, Telomerase prevents the accelerated cell ageing of Werner syndrome fibroblasts. Nat. Genet. 24, 16-17 (2000).

62. T. Matsumura, M. Nagata, R. Konishi, M. Goto, Studies of SV40-infected Werner syndrome fibroblasts. Adv. Exp. Med. Biol. 190, 313-330 (1985).

63. L. I. Huschtscha, K. V. A. Thompson, R. Holliday, The susceptibility of Werner's syndrome and other human skin fibroblasts to SV40-induced transformation and immortalization. Proc. R. Soc. London Ser. B 229, 1 $12(1986)$

64. H. Saito, R. E. Moses, Immortalization of Werner syndrome and progeria fibroblasts. Exp. Cell Res. 192, 373-379 (1991).

65. T. M. Rünger, C. Bauer, B. Dekant, K. Möller, P. Sobotta, C. Czerny, M. Poot, G. M. Martin, Hypermutable ligation of plasmid DNA ends in cells from patients with Werner syndrome. J. Invest. Dermatol. 102, 45-48 (1994).

66. D. M. J. Lilley, M. F. White, The junction-resolving enzymes. Nat. Rev. Mol. Cell Biol. 2, 433-443 (2001).

67. W.-D. Heyer, K. T. Ehmsen, J. A. Solinger, Holliday junctions in the eukaryotic nucleus: resolution in sight? Trends Biochem. Sci. 28, 548-557 (2003).

68. S. C. West, Processing of recombination intermediates by the RuvABC proteins. Annu. Rev. Genet. 31, 213-244 (1997).

69. A. Constantinou, M. Tarsounas, J. K. Karow, R. M. Brosh Jr., V. A. Bohr, I. D. Hickson, S. C. West, Werner's syndrome protein (WRN) migrates Holliday junctions and co-localizes with RPA upon replication arrest. EMBO Rep. 1, 80-84 (2000).

70. J. K. Karow, A. Constantinou, J.-L. Li, S. C. West, I. D. Hickson, The Bloom's syndrome gene product promotes branch migration of Holliday junctions. Proc. Natl. Acad. Sci. U.S.A. 97, 6504-6508 (2000).

71. A. Constantinou, A. A. Davies, S. C. West, Branch migration and Holliday junction resolution catalyzed by activities from mammalian cells. Cell 104 259-268 (2001).

72. A. Constantinou, X.-B. Chen, C. H. McGowan, S. C. West, Holliday junction resolution in human cells: two junction endonucleases with distinct substrate specificities. EMBO J. 21, 5577-5585 (2003).

73. N. M. Hollingsworth, S. J. Brill, The Mus81 solution to resolution: generating meiotic crossovers without Holliday junctions. Genes Dev. 18, 117-125 (2004).

74. X.-B. Chen, R. Melchionna, C.-M. Denis, P.-H. L. Gaillard, A. Blasina, I. V. de Weyer, M. N. Boddy, P. Russell, J. Vialard, C. H. McGowan, Human Mus81-associated endonuclease cleaves Holliday junctions in vitro. Mol. Cell 8, 1117-1127 (2001).

75. H. Gao, X.-B. Chen, C. H. McGowan, Mus81 endonuclease localizes to nucleoli and to regions of DNA damage in human S-phase cells. Mol. Biol. Cell 14, 4826-4834 (2003).

76. V. Blais, H. Gao, C. A. Elwell, M. N. Boddy, P.-H. L. Gaillard, P. Russell, C. H. McGowan. RNAi inhibition of Mus81 reduces mitotic recombination in human cells. Mol. Biol. Cell 15, 552-562 (2004).

77. J. Abraham, B. Lemmers, M. P. Hande, M. E. Moynahan, C. Chahwan, A Ciccia, J. Essers, K. Hanada, R. Chahwan, A. K. Khaw, et al., Eme1 is involved in DNA damage processing and maintenance of genomic stability in mammalian cells. EMBO J. 22, 6137-6147 (2003).

78. Y. Liu, J.-Y. Masson, S. Shah, P. O'Regan, S. C. West, RAD51C is required for Holliday junction processing in mammalian cells. Science $303,243-246$ (2004).

79. J. Thacker, M. Z. Zdzienicka, The mammalian XRCC genes: their roles in DNA repair and genetic stability. DNA Repair 2, 655-672 (2003).

80. M. Brenneman, B. M. Wagener, C. A. Miller, C. Allen, J. A. Nickoloff, XRCC3 controls the fidelity of homologous recombination: roles for XRCC3 in late stages of recombination. Mol. Cell 10, 387-395 (2002).

81. J. Henry-Mowatt, D. Jackson, J. Y. Masson, P. A. Johnson, P. M. Clements F. E. Benson, L. H. Thompson, S. Takeda, S. C. West, K. W. Caldecott, XRCC3 and Rad51 modulate replication fork progression on damages verte- 
brate chromosomes. Mol. Cell 11, 1109-1117 (2003)

82. G. Ira, A. Malkova, G. Liberi, M. Foiani, J. E. Haber, Srs2 and Sgs1-Top3 suppress crossovers during double-strand break repair in yeast. Cell 115, 401-411 (2003).

83. B. Rockmill, J. C. Fung, S. S. Branda, G. S. Roeder, The Sgs1 helicase regulates chromosome synapsis and meiotic crossing over. Curr. Biol. 13, 1954-1962 (2003).

84. L. Wu, I. D. Hickson, The Bloom's syndrome helicase suppresses crossing over during homologous recombination. Nature 426, 870-874 (2003).

85. F. G. Harmon, R. J. DiGate, S. C. Kowalczykowski, RecQ helicase and topoisomerase III comprise a novel DNA strand passage function: a conserved mechanism for control of DNA recombination. Mol. Cell 3, 611-620 (1999).

86. J. German, Bloom syndrome: a Mendelian prototype of somatic mutational disease. Medicine 72, 393-406 (1993).

87. B. Michel, Replication fork arrest and DNA recombination. Trends Biochem. Sci. 25, 173-178 (2000).

88. M. L. G. Dronkert, R. Kanaar, Repair of DNA interstrand cross-links. Mutat. Res. 486, 217-247 (2001).

89. J. Courcelle, P. C. Hanawalt, RecQ and RecJ process blocked replication forks prior to the resumption of replication in UV-irradiated Escherichia coli. Mol. Gen. Genet. 262, 543-551 (1999).

90. K. Baynton, M. Otterlei, M. Bjørås, C. von Kobbe, V. A. Bohr, E. Seeberg WRN interacts physically and functionally with the recombination mediator protein RAD52. J. Biol. Chem. 278, 36476-36486 (2003)

91. F. Fabre, A. Chan, W.-D. Heyer, S. Gangloff, Alternate pathways involving Sgs1/Top3, Mus81/Mms4, and Srs2 prevent formation of toxic recombination intermediates from single-stranded gaps created during DNA replication. Proc. Natl. Acad. Sci. U.S.A. 99, 16887-16892 (2002)

92. M. R. Lieber, Y. Ma, U. Pannicke, K. Schwarz, Mechanism and regulation of human non-homologous DNA end-joining. Nat. Rev. Mol. Cell Biol. 4, 712 720 (2003).

93. P. R. Prince, C. E. Ogburn, M. J. Moser, M. J. Emond, G. M. Martin, R. J. Monnat Jr., Cell fusion corrects the 4-nitroquinoline 1-oxide sensitivity of Werner syndrome fibroblast cell lines. Hum. Genet. 105, 132-138 (1999).

94. S. M. Yannone, S. Roy, D. W. Chan, M. B. Murphy, S. Huang, J. Campisi, D. J. Chen, Werner syndrome protein is regulated and phosphorylated by DNA-dependent protein kinase. J. Biol. Chem. 276, $38242-38248$ (2001).

95. M. Takata, M. S. Sasaki, E. Sonoda, C. Morrison, M. Hashimoto, H. Utsumi, Y. Yamaguchi-Iwai, A. Shinohara, S. Takeda, Homologous recombination and non-homologous end-joining pathways of DNA double-strand break repair have overlapping roles in the maintenance of chromosomal integrity in vertebrate cells. EMBO J.17, 5497-5508 (1998).

96. C. Richardson, M. Jasin, Coupled homologous and nonhomologous repair of a double-strand break preserves genome integrity in mammalian cells. Mol. Cell. Biol. 20, 9068-9075 (2000).

97. C. Allen, J. Halbrook, J. A. Nickoloff, Interactive competition between homologous recombination and non-homologous end joining. Mol. Cancer Res. 1, 913-920 (2003).

98. J. Prudden, J. S. Evans, S. P. Hussey, B. Deans, P. O'Neill, J. Thacker, T. Humphrey, Pathway utilization in response to a site-specific DNA doublestrand break in fission yeast. EMBO J. 22, 1419-1430 (2003).

99. M. Frank-Vaillant, S. Marcand, Transient stability of DNA ends allows nonhomologous end joining to recede homologous recombination. Mol. Cell 10, 1189-1199 (2003).
100. A. J. Pierce, P. Hu, M. Han, N. A. Ellis, M. Jasin, Ku DNA end-binding protein modulates homologous repair of double-strand breaks in mammalian cells. Genes Dev. 15, 3237-3242 (2001).

101. C. Lundin, K. Erixon, C. Arnaudeau, N. Schultz, D. Jenssen, M. Meuth, T. Helleday, Different roles for nonhomologous end joining and homologous recombination following replication arrest in mammalian cells. Mol. Cell. Biol. 22, 5869-5878 (2002).

102. K. Fukuchi, G. M. Martin, R. J. Monnat Jr., Mutator phenotype of Werner syndrome is characterized by extensive deletions. Proc. Natl. Acad. Sci. U.S.A. 86, 5893-5897 (1989).

103. R. J. Monnat Jr., A. F. M. Hackmann, T. A. Chiaverotti, Nucleotide sequence analysis of human hypoxanthine phosphoribosyltransferase gene deletions. Genomics 13, 777-787 (1992).

104. R. J. Monnat Jr., in From Premature Gray Hair to Helicase-Werner Syndrome: Implications for Aging and Cancer, M. Goto, R. W. Miller, Eds. (Japan Scientific Societies Press, Tokyo, Japan, 2001), pp. 83-94.

105. F. H. Gage, J. Neurosci. 22, 612-643 (2004).

106. M. A. Nowak, F. Michor, Y. Iwasa, The linear process of somatic evolution. Proc. Natl. Acad. Sci. U.S.A. 100, 14966-14969 (2003).

107. M. J. Moser, W. L. Bigbee, S. G. Grant, M. J. Emond, R. G. Langlois, R. H. Jensen, J. Oshima, R. J. Monnat Jr., Genetic instability and hematologic disease risk in Werner syndrome patients and heterozygotes. Cancer Res. 60, 2492-2496 (2000).

108. R. S. Houlston, I. P. M. Tomlinson, Detecting low penetrance genes in cancer: the way ahead. J. Med. Genet. 37, 161-167 (2000).

109. M. Goto, R. W. Miller, Y. Ishikawa, H. Sugano, Excess of rare cancers in Werner syndrome (adult progeria). Cancer Epidemiol. Biomarkers Prev. 5, 239-246 (1996).

110. R. J. Monnat Jr., in World Health Organization Classification of Tumours. Pathology and Genetics of Tumors of Soft Tissue and Bone, C. Fletcher, K. Unni, F. Mertens, Eds. (IARC Press, Lyon, France, 2002), pp. 273-274.

111. M. Okada, M. Goto, Y. Furuichi, M. Sugimoto, Differential effects of cytotoxic drugs on mortal and immortalized B-lymphoblastoid cell lines from normal and Werner's syndrome patients. Biol. Pharm. Bull. 21, 235-239 (1998).

112. L. Ye, T. Miki, J. Nakura, J. Oshima, K. Kamino, H. Rakugi, H. Ikegami, J. Higaki, S. D. Edland, G. M. Martin, et al., Association of a polymorphic variant of the Werner helicase gene with myocardial infarction in a Japanese population. Am. J. Hum. Genet. 68, 494-498 (1997).

113. E. Castro, C. E. Ogburn, K. E. Hunt, R. Tilvis, J. Louhija, R. Penttinen, R. Erkkola, A. Panduro, R. Riestra, C. Piussan, et al., Polymorphisms at the Werner locus: I. Newly identified polymorphisms, ethnic variability of 1367Cys/Arg, and its stability in a population of Finnish centenarians. Am. J. Med. Genet. 82, 399-403 (1999).

114. E. Castro, S. D. Edland, L. Lee, C. E. Ogburn, S. S. Deeb, G. Brown, A. Panduro, R. Riestra, R. Tilvis, J. Louhija, et al., Polymorphisms at the Werner locus: II. 1074Leu/Phe, 1367Cys/Arg, longevity, and atherosclerosis. Am. J. Med. Genet. 95, 374-380 (2000).

115. G. Passarino, P. Shen, J. B. Van Kirk, A. A. Lin, G. De Benedictis, L. L. Cavalli Sforza, P. J. Oefner, P. A. Underhill, The Werner syndrome gene and global sequence variation. Genomics 71, 118-122 (2001).

116. L. S. Symington, Role of RAD52 epistasis group gene in homologous recombination and double-strand break repair. Microbiol. Mol. Biol. Rev. 66, 630-670 (2002). 